\title{
Planetary nebulae and determination of the bulge--disk boundary
}

\author{
Roberto D. D. Costa, Oscar Cavichia, and Walter J. Maciel \\ Departamento de Astronomia, IAG, Universidade de São Paulo, \\ Rua do Matão 1226, 05508-090, São Paulo/SP, Brazil \\ email: [roberto, cavichia, maciel] @astro.iag.usp.br
}

\begin{abstract}
In this paper, a sample of planetary nebulae in the Galaxy's inner-disk and bulge is used to find the galactocentric distance that optimally separates these two populations in terms of their abundances. Statistical distance scales were used to investigate the distribution of abundances across the disk-bulge interface, while a Kolmogorov-Smirnov test was used to find the distance at which the chemical properties of these regions separate optimally. The statistical analysis indicates that, on average, the inner population is characterized by lower abundances than the outer component. Additionally, for the $\alpha$-element abundances, the inner population does not follow the disk's radial gradient toward the Galactic Center. Based on our results, we suggest a bulge-disk interface at $1.5 \mathrm{kpc}$, marking the transition between the bulge and the inner disk of the Galaxy as defined by the intermediate-mass population.
\end{abstract}

Keywords. techniques: spectroscopic, methods: numerical, planetary nebulae: general, Galaxy: structure

\section{Introduction}

Distances to planetary nebulae (PNe) are poorly known in Galactic astronomy, since there is not a single observational parameter that is directly related to their distances. The usual procedure is to adopt statistical distance scales to estimate distances to individual objects. On the other hand, abundances of PNe in the inner disk and bulge provide important information about the concentration of elements such as helium (He), nitrogen $(\mathrm{N})$, oxygen $(\mathrm{O})$, sulfur $(\mathrm{S})$, argon $(\mathrm{Ar})$, and neon $(\mathrm{Ne})$ in this region. Since PNe originate from progenitor stars with initial masses from 0.8 to $8 \mathrm{M}_{\odot}$, the abundances of their $\alpha$ elements reflect the chemical evolution of the interstellar medium over a large fraction of the Galaxy's lifetime. Therefore, these parameters, associated with the evolution of intermediate-mass stars, become important constraints to model the chemical evolution of the Galaxy.

Focusing on PNe, many studies - including Górny et al. (2004) and Gutenkunst et al. (2008) - reveal a discontinuity in the abundance gradient toward the Galactic Center, in the sense that the abundances of bulge PNe do not follow the same trend as disk PNe. The radial gradient of the $\mathrm{O}$ abundance derived from the PN population in the Galactic disk flattens in the innermost regions of the Galaxy, and may even change sign.

\section{Observations and data reduction}

The observational data were obtained using the $1.6 \mathrm{~m}$ telescope at the Pico dos Dias Observatory/MCT (Brazilian Ministry of Science and Technology). To select a sample of $\mathrm{PNe}$ in the bulge or inner disk, we singled out PNe with $5 \mathrm{GHz}$ fluxes $<100 \mathrm{mJy}$, optical diameters $<12^{\prime \prime}$, and Galactic coordinates within $10^{\circ}$ from the position of the Galactic Center. Combination of these criteria leads to rejection of approximately $90-95 \%$ of $\mathrm{PNe}$ 
projected in the Galactic Center direction, but which have heliocentric distances $<4$ kpc.

Data reduction was performed in the standard way for long-slit spectroscopy using IRAF tools. Electron densities were derived from the [SII] $\lambda 6716 \AA / \lambda 6731 \AA$ line ratio, electron temperatures from the [OIII] $\lambda 5007 \AA / \lambda 4363 \AA$ and $[\mathrm{NII}] \lambda 6581 \AA / \lambda 5754 \AA$ line ratios, and ionic abundances were calculated using the equations of Alexander \& Balick (1997). Helium abundances were derived from recombination theory, with corrections for collisional excitation. N, O, S, Ar, and Ne elemental abundances were calculated by adopting the ionization correction factors of Escudero et al. (2004). The sample, observations, and data reduction are described in detail in Cavichia et al. (2010).

\section{The bulge--disk boundary}

To investigate the distance distributions of bulge and inner-disk $\mathrm{PNe}$, we used statistical distance scales. A large number of such scales have been proposed in the literature, and we adopted two to study the chemical abundance distribution, i.e., the Stanghellini et al. (2008) - hereinafter SSV08 - and the Zhang (1995) - henceforth Z95 - scales. Both are based on the Shklovsky method, but with the addition of a relationship between ionized masses and radii of $\mathrm{PNe}$.

The distance distribution of the chemical abundances and the determination of the bulge-disk interface were investigated by employing a method similar to that suggested by Maciel et al. (2006). First, a given galactocentric distance $\left(R_{\mathrm{L}}\right)$ is chosen, which defines a limit for the sample. Next, the sample is divided into two groups. Group I is composed of $\mathrm{PNe}$ at galactocentric distances smaller than $R_{\mathrm{L}}$, and group II consists of PNe at greater galactocentric distances. For each group, the average abundances are calculated for the available elements. Then, the limiting radius $R_{\mathrm{L}}$ is varied from 0.1 to $3.6 \mathrm{kpc}$ in steps of $0.7 \mathrm{kpc}$, for both distance scales. Fig. 1 illustrates the results for the SSV08 and Z95 scales. In each plot, group I is represented by filled circles connected by continuous lines and group II by squares joined by dashed lines. Each pair of circles/squares in each plot represents the average abundance for that element as a function of the limiting distance, adopting a given limit for the bulge-disk interface. Therefore, each plot shows
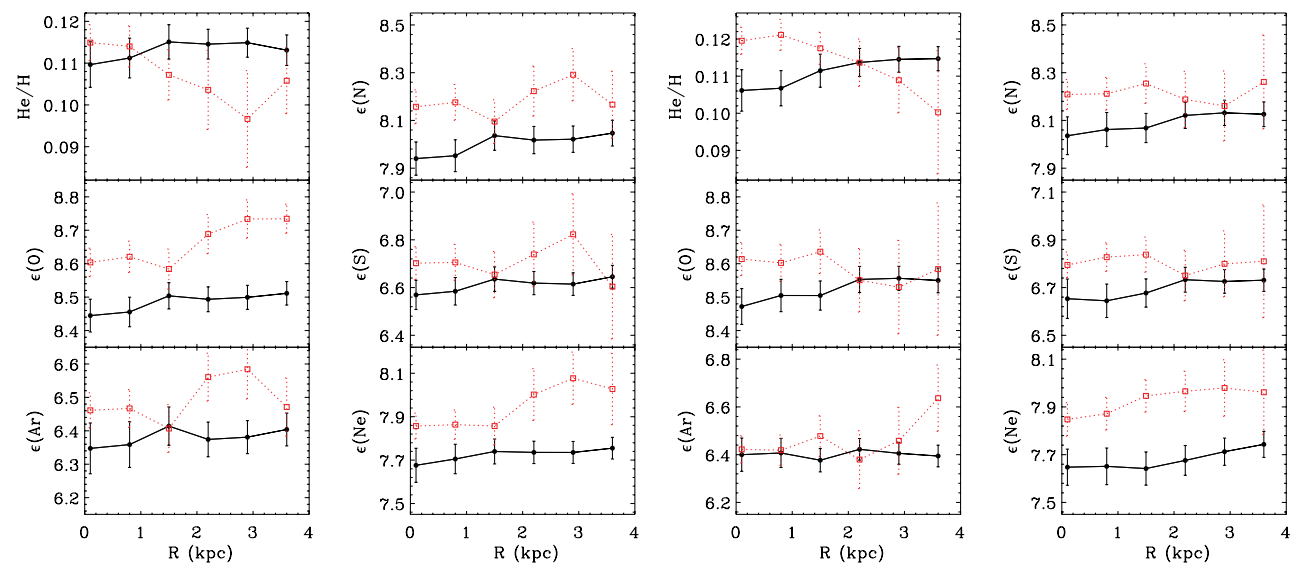

Figure 1. Average radial distribution of PNe chemical abundances based on (left) the Stanghellini et al. (2008; SSV08) and (right) the Zhang (1995; Z95) distance scales. Black solid circles represent group I and red open squares represent group II PNe. For the Z95 scale, $R$ is given as $R_{\mathrm{L}}$. 
Table 1. Average abundances of groups I and II for both distance scales.

\begin{tabular}{lcccc}
\hline & \multicolumn{2}{c}{ SSV08 } & \multicolumn{2}{c}{ Z95 } \\
\hline Element & Group I & Group II & Group I & Group II \\
\hline $\mathrm{He} / \mathrm{H}$ & $0.110 \pm 0.004$ & $0.099 \pm 0.009$ & $0.111 \pm 0.004$ & $0.117 \pm 0.004$ \\
$\epsilon(\mathrm{N})$ & $8.02 \pm 0.06$ & $8.22 \pm 0.10$ & $8.07 \pm 0.06$ & $8.25 \pm 0.08$ \\
$\epsilon(\mathrm{O})$ & $8.49 \pm 0.04$ & $8.69 \pm 0.06$ & $8.50 \pm 0.04$ & $8.64 \pm 0.07$ \\
$\epsilon(\mathrm{S})$ & $6.62 \pm 0.05$ & $6.74 \pm 0.14$ & $6.68 \pm 0.06$ & $6.84 \pm 0.07$ \\
$\epsilon(\mathrm{Ar})$ & $6.37 \pm 0.05$ & $6.56 \pm 0.07$ & $6.38 \pm 0.05$ & $6.48 \pm 0.08$ \\
$\epsilon(\mathrm{Ne})$ & $7.74 \pm 0.05$ & $8.00 \pm 0.12$ & $7.64 \pm 0.07$ & $7.95 \pm 0.07$ \\
\hline
\end{tabular}

how the differences between the groups evolve when adopting distinct limits. As expected, the results are inconclusive for $\mathrm{He}$ or $\mathrm{N}$, since the abundances of these elements change during the evolution of the progenitor stars. Irrespective of the distance scale adopted, it can be seen that for the $\alpha$ elements the differences between both samples attain a minimum between $1.5 \mathrm{kpc}$ and $2.5 \mathrm{kpc}$.

To determine a value for the galactocentric distance of the bulge-disk interface based on the intermediate-mass population represented by the $\mathrm{PNe}$ sample, we performed Kolmogorov-Smirnov tests for groups I and II during each step of our adopted procedure. The tests return the probabilities that both groups are drawn from the same distribution. Low probabilities imply that the cumulative distribution function of group I is significantly different from that of group II, and that they hence represent different populations. The test results are shown in Fig. 2, where the filled histograms correspond to the SSV08 scale and the open histograms to the Z95 scale. The results clearly indicate that a minimum $\mathrm{K}-\mathrm{S}$ probability occurs for the $\mathrm{Z} 95$ scale, at $R \sim 1.5 \mathrm{kpc}$, but also that for the SSV08 scale the position of any minimum is not as clear.

Upon adoption of the galactocentric distance that optimally separates the disk and bulge characteristics, the average abundances were calculated for both groups, adopting distances of 1.5 and $2.2 \mathrm{kpc}$ for, respectively, the Z95 and SSV08 scales as limits. The results are listed in Table 1. Although these abundances are similar, reflecting the large dispersion in abundances found in these regions of the Galaxy, some important differences

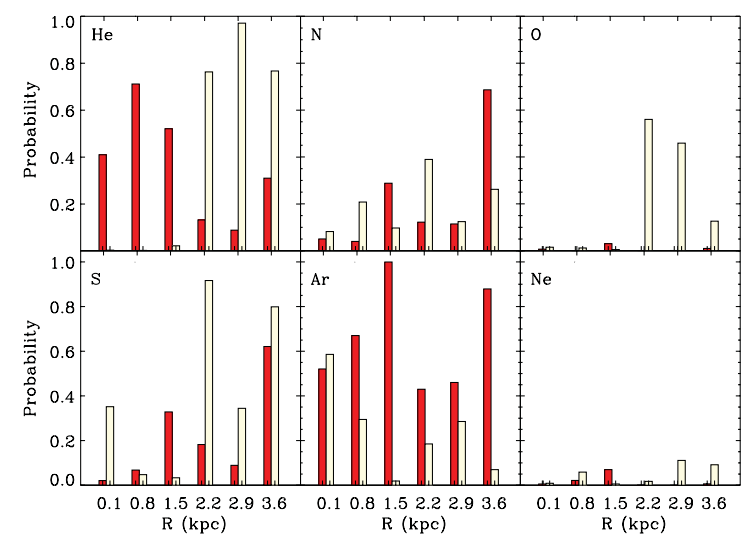

Figure 2. Kolmogorov-Smirnov $(\mathrm{K}-\mathrm{S})$ probability histogram for all elements as a function of the group I/II separation radius. The solid (red) and open (yellow) histograms correspond to the SSV08 and Z95 scales, respectively. The results clearly indicate that a minimum $\mathrm{K}-\mathrm{S}$ probability occurs for the Z95 scale at $R \sim 1.5 \mathrm{kpc}$. However, for the SSV08 scale the position of any minimum is not as clear. 
are apparent. In particular, the N, O, S, Ar, and Ne abundances are lower for group I than for group II for both distance scales. For $\mathrm{N}$ and $\mathrm{O}$, the difference between the two groups is 0.20 dex. For $\mathrm{S}, \mathrm{Ar}$, and Ne it is $0.12,0.19$, and 0.26 dex, respectively. On average, abundances of objects whose galactocentric distances place them in the bulge and not in the inner disk according to either distance scale are lower than those of objects with distances in excess of this limit. However, this difference is not larger than the errors in individual abundances. Considering the SSV08 distance scale, the abundance difference between the two groups of PNe is based on 68,62, 53, and $56 \mathrm{PNe}$ of group I (bulge) for $\mathrm{O}, \mathrm{S}, \mathrm{Ar}$, and $\mathrm{Ne}$, respectively, and $16,15,14$, and $12 \mathrm{PNe}$, respectively, of group II (inner disk) for the same elements. For the Z95 distance scale, the abundance difference is based on, respectively, 72, 66, 50, and 43 PNe of group I, and 41, 39, 28, and 29 of Group II, again for the same elements. Figure 3 shows these distributions for both groups.

Taking into account the results derived in this paper, as well as other evidence from the literature, we propose a galactocentric distance of $1.5 \mathrm{kpc}$ (based on the Z95 distance scale) as the transition between the bulge and the inner disk of the Galaxy. A more detailed description of this work can be found in Cavichia et al. (2011).
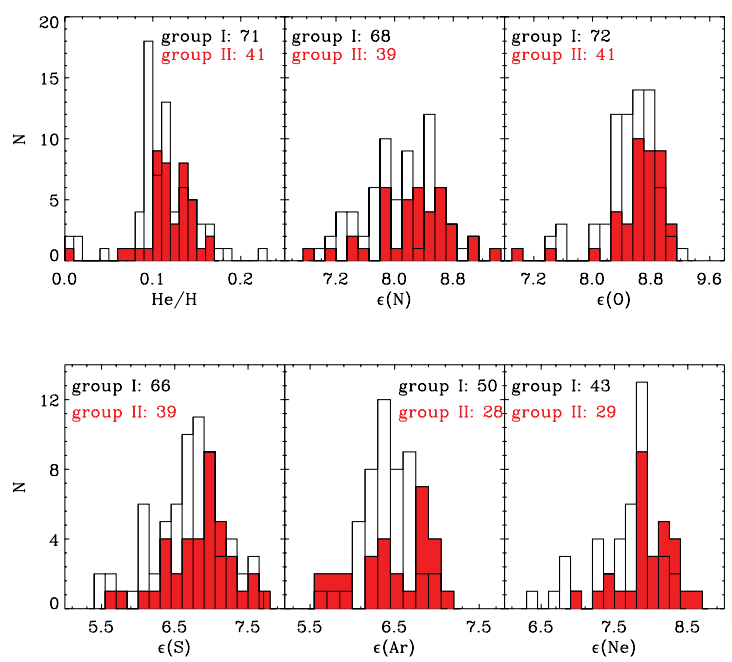

Figure 3. Abundance distributions for groups I and II using the Z95 scale (left; $R=1.5 \mathrm{kpc}$ ). Open histograms represent group I objects and filled histograms are for group II. The number of objects contained in each distribution is listed in the panel legends.

\section{References}

Alexander, J. \& Balick, B. 1997, AJ, 114, 713

Cavichia, O., Costa, R. D. D., \& Maciel, W. J. 2010, Rev. Mex. A\&GA, 46, 159

Cavichia, O., Costa, R. D. D., \& Maciel, W. J. 2011, Rev. Mex. A\&A, 47, 49

Escudero, A. V., Costa, R. D. D., \& Maciel, W. J. 2004, A\&A, 414, 211

Górny, S. K., Stasińska, G., Escudero, A. V., \& Costa, R. D. D. 2004, A\&̛A, 427, 231

Gutenkunst, S., Bernard-Salas, J., Pottasch, S., Sloan, G., \& Houck, J. R. 2008, ApJ, 680, 1206

Maciel, W. J., Lago, L., \& Costa, R. D. D. 2006, A\& $A, 453,587$

Stanghellini, L., Shaw, R. A., \& Villaver, E. 2008, ApJ, 689, 194 (SSV08)

Zhang, C. Y. 1995, ApJS, 98, 659 (Z95) 